\title{
Anomalous Flow Behavior in Closed and Open Thin Walled Nanochannels
}

Sohail Murad ${ }^{*}$ and Ishwar K. Puri ${ }^{\dagger}$

*Department of Chemical Engineering, University of Illinois at Chicago, Chicago, Illinois 60607, USA and ${ }^{\dagger}$ Department of Engineering Science and Mechanics, Virginia Polytechnic Institute and State University, Blacksburg, Virginia 24061, USA

Molecular dynamics simulations have been carried out to examine water flow in symmetric and asymmetric open and closed ends nanochannels with hydrophilic surfaces. The results are counterintuitive and the opposite of what is observed in macro-systems--closed channels fill faster with fluid than do their open counterparts. In addition, hybrid closed-open asymmetric channels fill up even faster. These results can be explained on the basis of the fluid-structure interaction that arises through the different vibrational behaviors of the surface molecules that are part of the wall forming these channels. Such effects are not expected to be of significance in macro-channels, and point to an important case where macro and nano-channels exhibit contrary behavior. Since these effects results from strong interactions between the fluid molecules and solid surface, one would not expect them with hydrophobic walls, and our simulations confirm such behavior.

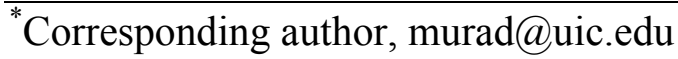


Fluid confinement and flow ${ }^{1,2}$ in nanoscale channels occurs often in nature, e.g., in cell membranes during ion and water transport ${ }^{3}$, and in rocks ${ }^{4}$. The physical properties of nanoscale water are known to differ from those of its bulk counterpart ${ }^{5}$. Studies on carbon nanotubes and hydrophobic surfaces have shown that the flow is virtually frictionless ${ }^{6,7}$, being limited primarily by the barriers at the entry and exit of the nanotube pore ${ }^{8}$, indicating that the structure outside a nanochannel has a significant influence on the flow within it ${ }^{9}$. On the other hand with hydrophilic surfaces, the flow experience considerable resistance to flow in the nanochannel ${ }^{7}$. We consider water flow in hydrophilic nanochannels that are constructed in various configurations through molecular dynamics (MD) simulations ${ }^{10,11}$ and show that the fluidstructure interaction arising through different vibrational behaviors of hydrophilic surface molecules bounding nanochannels can have a profound influence in driving water flow. Most surfaces will have surface thermal fluctuations, so the effects we are observing may affect a whole range of nanopores/nanoslits in nature. While natural nanpores can have various shapes and configurations that could couple fluid behavior with the free and driven vibrations of solid surfaces $^{12,13,14}$, the influences of geometry and the corresponding fluid-structure interactions on fluid flow have remained unexplored beyond interfacial issues such as fluid slip ${ }^{15}$, and hydrophobic or hydrophilic interactions. ${ }^{16}$ A better understanding of such behavior ${ }^{17}$ would help in the development nanoscale devices from a more fundamental perspective than simply mimicking macroscale design methodologies.

The MD simulations nominally consider 3968-3984 molecules in the basic cyclically replicated parallelepiped shown in Fig. 1. The system dimensions are $L_{x}=11.2 \mathrm{~nm}$ axially, and $\mathrm{L}_{\mathrm{z}}=\mathrm{L}_{\mathrm{z}}=3.73 \mathrm{~nm}$ in each transverse direction. As shown in Fig. 1, two moveable walls uniformly compress fluid reservoirs, forcing fluid into $0.5 \mathrm{~nm}$ wide and infinitely deep channels that are 
initially evacuated. These channels are constructed in the four configurations shown in Fig. 2, namely, continuous (Type 1), open-open (Type 2), closed-closed (Type 3), and closed-open (Type 4). The solid walls are modeled after Si and have 2096-2112 sites, depending upon the configuration. The Si molecules are tethered to their equilibrium sites with a simple harmonic potential with a spring constant of $44.7 \mathrm{~N} / \mathrm{m}$. These walls enclose 1872 water molecules. All molecules are provided with initial Gaussian velocity distributions. Both the fluid and the wall temperatures are maintained at $334 \mathrm{~K}$ using a Gaussian thermostat. The simulated results do not change if other techniques, such as simple velocity rescaling, are used.

The simulation algorithm uses the quaternion method with a fifth-order Gear predictorcorrector algorithm for translational motion and a fourth-order predictor-corrector algorithm for rotational motion. ${ }^{18,19}$ Intermolecular interactions are described by the potential model $u_{i j}=4 \varepsilon_{i j}\left(\left(\sigma_{i j} / r_{i j}\right)^{12}-\left(\sigma_{i j} / r_{i j}\right)^{6}\right)+q_{i} q_{j} / r_{i j}$, where $\sigma_{i j}$ and $\varepsilon_{i j}$ denote the LJ interaction parameters, $r_{i j}$ the scalar distance between sites $\mathrm{i}$ and $\mathrm{j}$, and $q_{i}$ and $q_{j}$ charges on sites when applicable. Cross interactions are modeled based on Lorentz-Berthelot mixing rules ${ }^{11}$ and the reaction field method is used for long-range interactions. ${ }^{20,21}$ The water parameters $\sigma$ and $\varepsilon$, presented in Table 1, represent experimental geometries, energies of ion-water complexes, and energies of solvation $^{22}$ for water, which is modeled after the SPC potential ${ }^{23}$. Wall atoms are modeled as LJ sites. The hydrophilic interaction between water and the wall surfaces is controlled by placing random or systematic negative and positive charges on the wall atoms (or sites) while maintaining overall charge neutrality ${ }^{16,24,25}$. The atoms that constitute the nanochannel and walls are tethered using a simple harmonic potential. If some of these tethering sites are selectively moved, the walls are also dragged along (as illustrated in Fig. 1) while allowing for thermal 
fluctuations. A typical simulation runs for either 1,500,000 steps or until the channel fills up, whichever occurs first, with 0.176 femtosecond time steps.

Figure 2 presents images of fluid flow though the four channels after 500,000 time steps, while Figure 3 shows the fluid penetration for a range of time steps to provide a more detailed picture of the differences in the flow rates in these channels. These figures show that continuous and open-open channels offer far more resistance to fluid flow than do closed-closed and closedopen channels. The shortest fill time occurs for Type 4 closed-open asymmetric channels, which is counterintuitive. These results are the opposite of what one would expect in macro-channels. However, it should be noted that most macro-channels are usually initially filled with air. In our studies we evacuated the channels, but did allow for the fluid to evaporate in the channels naturally as determined by the thermodynamics of the system. In our system, since the channels are evacuated, the pressure gradients at the leading edges of the flows are identical in all four cases. The more obvious expectation was that the flow rate in all channels would be equal, since we saw little evidence of evaporation of the fluid during the simulation times, and in any case no evaporated fluids actually left the channel (thus the system would not be effected by the open/closed status of the channel via the fluid-fluid interactions at least). Thus the only possible explanation would be the fluid-solid interactions. The interaction parameters between the surface-water were identical, so the only possible explanation must lie with the "mechanical" behavior of the solid surface itself. We therefore hypothesized that the difference arises not from distinctions in the inherent fluid mechanics, but from the dissimilar fluid-structure interactions for the four cases. Thus the only remaining obvious difference could be due to the vibrational state of the surface molecules. The rational behind these differences is that the molecular plates bounding the channels are cantilevers that have different vibrational modes, since the plates 
bounding the Type 1 continuous channels are clamped at both ends, those for the Type 2 openopen symmetric channels are clamped at one and free at the other, clamped at one end but spring coupled at the other for the Type 3 closed-closed symmetric channels, and arranged in a hybrid clamped-spring coupled and clamped-free configuration for the Type 4 closed-open asymmetric channels ${ }^{26} \cdot{ }^{17}$ A free cantilever end essentially corresponds to one attached to an infinitely soft spring. These arrangements are schematically described in Fig. 2.

To understand the vibrational state of the surface molecules, the upper walls of the nanochannels were divided into eleven strips, ten of which, numbered 1-5 and 7-11 on each side, are typically two molecules wide in the axial direction and eight molecules deep in our configuration. However, due to the periodic boundary conditions the strips are effectively of infinite width. An eleventh central strip, denoted as 6 , is ignored since it corresponds to the void in the upper wall that lies in the middle of the domain for the Type 2-4 configurations. The cantilever vibrations are monitored through the strip displacements $y(t)$ (perpendicular to the wall surface) as the nanochannels are filled with fluid. The displacements of strips 2-5 are presented in Figs. 4(a) and (b) for two configurations, i.e., the continuous channels in Fig. 4(a) and the closed-open channels in Fig. 4(b). While each strip exhibits periodic oscillations, these vibrations are disorganized for the Type 1 (and Type 2 and 3) configurations but are correlated in case of the Type 4 closed-open nanochannels. The displacements of strips 6-9 for the Type 4 open nanochannel are also correlated, as shown in Fig. 4(c). The strip vibration amplitudes are smallest for the Type 1 continuous nanochannel, explaining why fluid flow into it is also slowest. Figure 4(d) presents the correlations coefficients $c_{i, 2}$ and $c_{j, 10}$, where $\mathrm{i}=3,4,5$ and $\mathrm{j}=7,8,9$, for the displacements for all four configurations. These are strongly correlated for the Type 4 configuration, but exhibit weak correlations for the other three cases. The correlations are also 
stronger for Type 3 (closed-closed) which also shows significantly larger flow rate than Types 1 and 2 (continuous and open-open)

A free cantilever end (at $x=L$ ) has a larger vibration amplitude, since $d^{2} y / d x^{2}=d^{3} y / d x^{3}=0$, than one attached to a stiff spring, which mimics a pinned end with $y \approx d^{2} y / d x^{2} \approx 0 .{ }^{17}$ Hence, while the maximum vibration amplitude occurs at $x=L$ for a cantilever with a free end, this is not the case for one attached to a stiff spring. Noting that the strip displacements are attributable to a combination of vibration modes, we surmise from Figs. $2-4$ that cantilevers with ends attached to relatively stiff springs have correlated movements along their lengths, which allow the nanochannels established between them to fill more quickly than those formed between cantilevers with free ends. Our conjecture is that filling of the open nanochannel in the Type 4 configuration influences the reservoir dynamics, which then drive the filling of the corresponding open nanochannel. This coupled dynamic likely leads to an optimization of the spring constant associated with the lattice molecules connecting the top and bottom molecular plates of the closed nanochannel. Superimposition of the various vibration modes leads to more favorable deformations along their length, which allows the Type 4 closed nanochannel to fill more quickly than in the other configurations.

When the wall surface is made hydrophobic, all configurations essentially exhibit the same flow behavior, i.e., the fluid-structure interactions no longer influence the filling of the nanochannels. This is unsurprising, since water molecules have minimal interactions with hydrophobic surface molecules. Thus, the vibrations of the bounding cantilever surface should not be expected to influence the local flow behavior. The simulations reveal an interesting effect that is only of consequence in nanoscale systems, i.e., fluid flow in these small systems can be profoundly influenced by the vibrational characteristics of hydrophilic bounding surface 
molecules. We are not aware of previous results that have pointed out such anomalous behavior, either theoretically or experimentally.

\section{Acknowledgements}

This research was supported in part by a grant from the Office of Basic Energy Science, Department of Energy [Grant No. DE-FG02-08ER46538]. 


\section{List of Figures}

Figure 1: Schematic diagram of the domain for an arbitrary simulation where $L_{x}=11.2 \mathrm{~nm}$, and $L_{y}=L_{z}=3.73 \mathrm{~nm}$. The hydrophilic walls are moved in the directions shown to compress the fluid into channels that have different end configurations, as shown in Fig. 2.

Figure 2: Fluid penetration into evacuated channels after 500,000 time steps, each of 0.176 fs duration, for four different channel configurations: (a) continuous, (b) open-open, (c) closedclosed, and (d) closed-open. The few water molecules seen outside the walls leak through the tethered wall molecules at high pressures.

Figure 3: Results for fluid penetration in the channels at intervals of 125,000 time steps each of length 0.18 fs.

Figure 4: Movements of strips 2-5 for two channel configurations: (a) continuous, (b) closedopen (strips 2-5), and closed-open (strips 7-10). (d) The correlation coefficients $\mathrm{c}_{\mathrm{i}, 2}$ and $\mathrm{cj}_{, 10}$, with $\mathrm{i}=3,4,5$ and $\mathrm{j}=7,8,9$, for the strip locations for all four configurations presented in Fig. 2 . The 81 results in (a)-(c) are presented after every 2500 time steps, with each step of $1.76 \times 10^{-16} \mathrm{~s}$ duration. The positions are scaled by $0.19 \mathrm{~nm}$. Thus, a location corresponding to 11 dimensionless units corresponds to $2.09 \mathrm{~nm}$. 


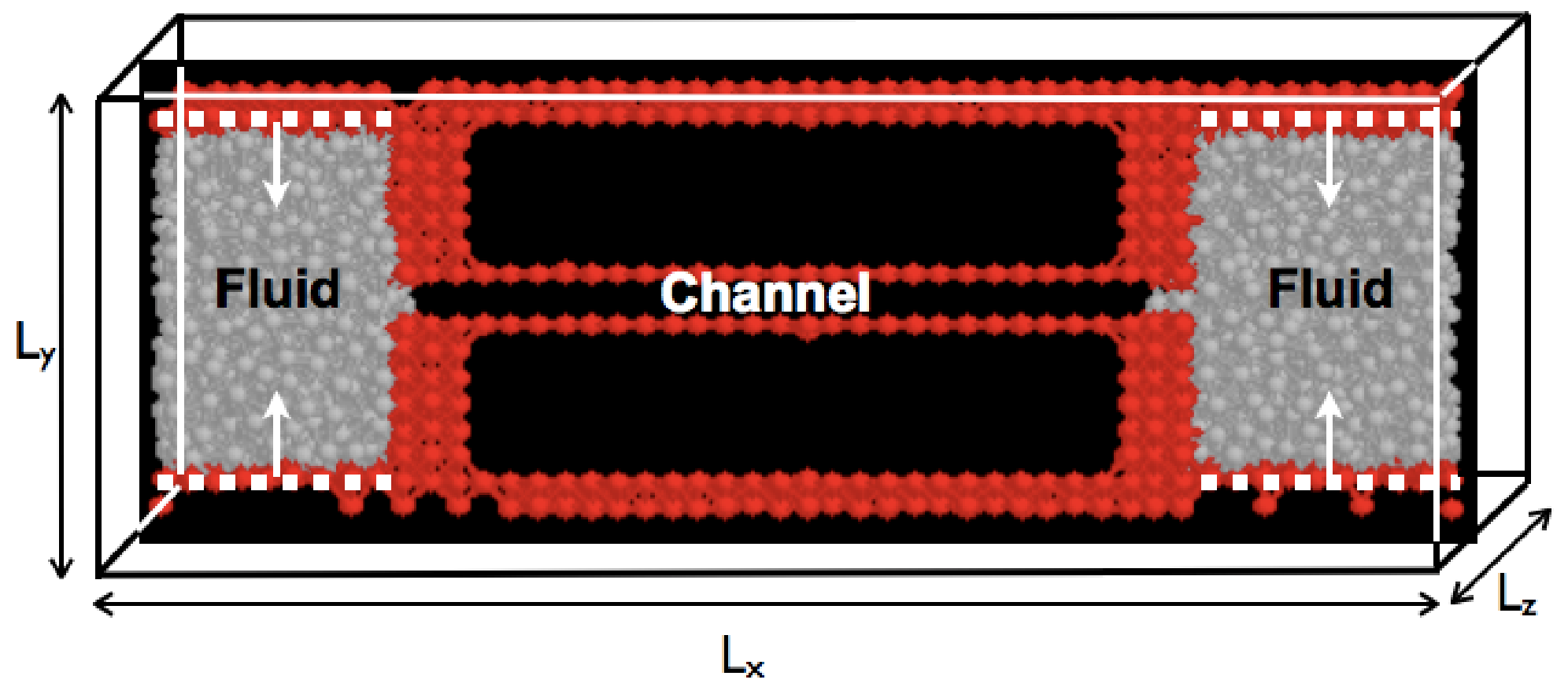



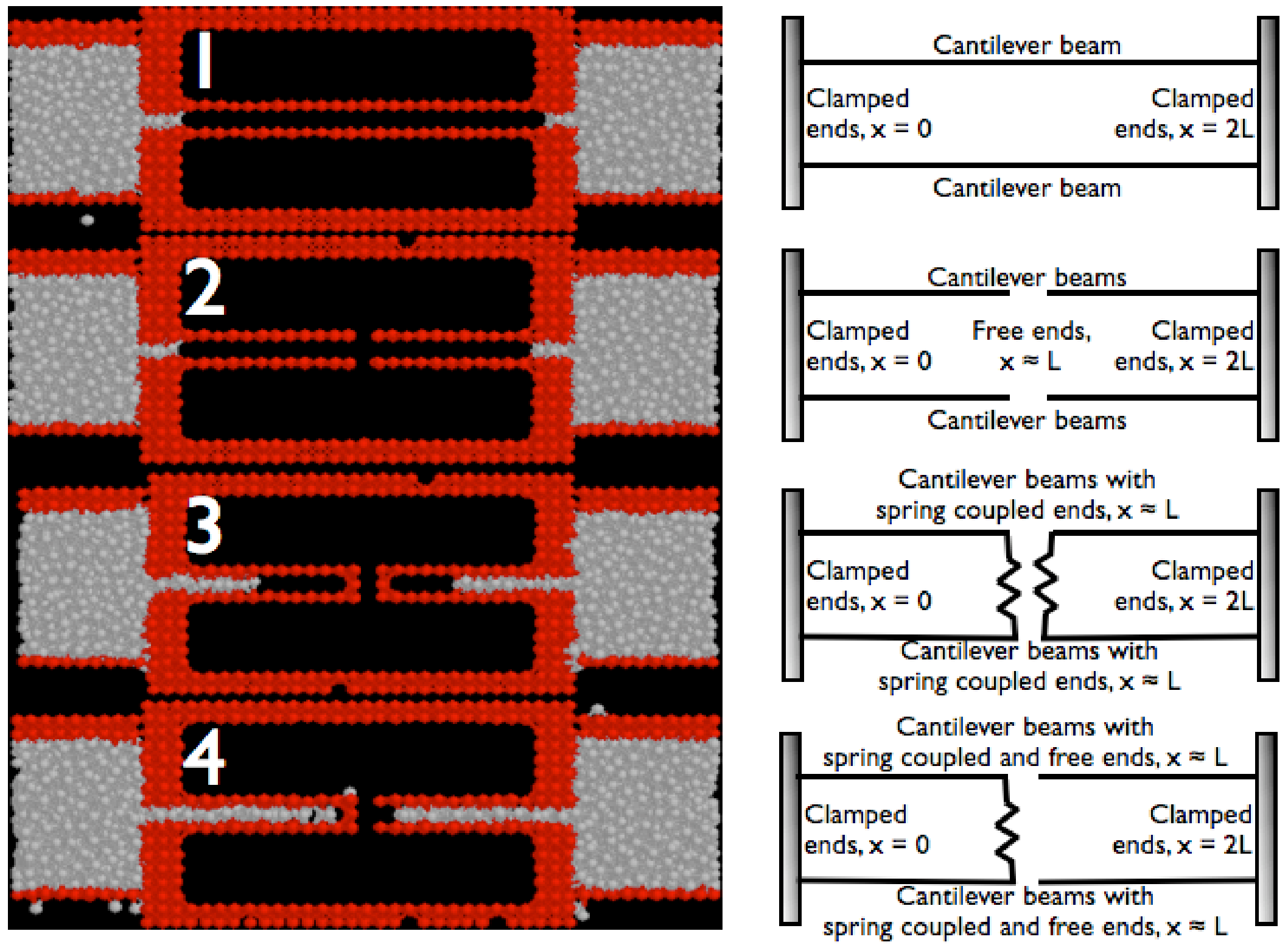


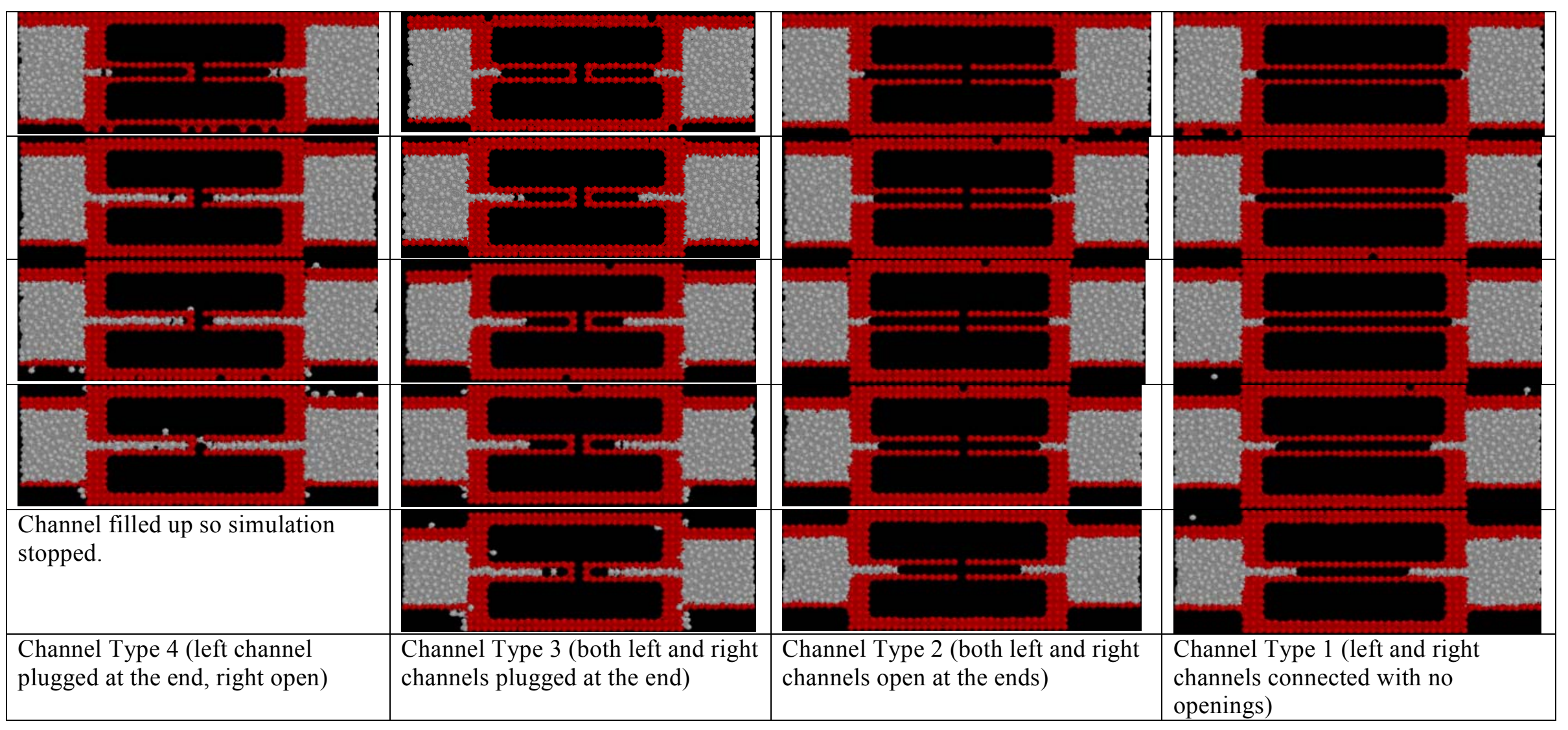







Table I: Values of the Lennard-Jones interactions parameters $\sigma, \varepsilon$, and $q$. Cross interactions are modeled based on Lorentz-Berthelot mixing rules.

\begin{tabular}{cccc}
\hline & $\sigma$ & $\varepsilon$ & $q$ \\
& $10^{-10} \mathrm{~m}$ & $\mathrm{KJ} \mathrm{mol}^{-1}$ & $\mathrm{e}$ \\
\hline $\mathrm{O}$ & 3.17 & 0.65 & -0.82 \\
$\mathrm{H}$ & 0 & 0 & 0.41 \\
$\mathrm{Wall}$ & 4.028 & 0.87 & 0 \\
\hline
\end{tabular}




\section{$\underline{\text { References }}$}

1 A. D. Stroock, S. K. Dertinger, Ajdari, A. Ajdari, I.Mezic, H. A Stone, G. M. Whitesides, "Chaotic Mixer for Microchannels" Science, 295, 647-654 (2002).

2 H. Kitano, “Systems Biology: A Brief Overview”, Science, 295, 1662-1664 (2002)

3 P. Agre, "Aquaporin Water Channels (Nobel Lecture)," Angewandte Chemie International Edition 43 (33), 4278-4290 (2004).

4 Y. F. Wang, C. Bryan, H. F. Xu et al., "Nanogeochemistry: Geochemical reactions and mass transfers in nanopores," Geology 31 (5), 387-390 (2003).

3 A. I. Kolesnikov, J. M. Zanotti, C. K. Loong et al., "Anomalously soft dynamics of water in a nanotube: A revelation of nanoscale confinement," Phys. Rev. Lett. 93 (3), 4 (2004).

6 M. Majumder, N. Chopra, R. Andrews et al., "Nanoscale hydrodynamics - Enhanced flow in carbon nanotubes," Nature 438 (7064), 44-44 (2005).

7 S. Murad, L. Luo and L-Y Chu, "Anomalous Flow Behavior in Nanochannels: A Molecular Dynamics Study", Chemical Physics Letters, in press (2010)

8 A. Kalra, S. Garde, and G. Hummer, "Osmotic water transport through carbon nanotube membranes," Proc. Natl. Acad. Sci. U. S. A. 100 (18), 10175-10180 (2003).

9 X. J. Gong, J. Y. Li, H. Zhang et al., "Enhancement of Water Permeation across a Nanochannel by the Structure outside the Channel," Phys. Rev. Lett. 101 (25) (2008).

10 S. Murad and J. Lin, "Molecular modeling of fluid separations using membranes: effect of molecular forces on mass transfer rates," Chemical Engineering Journal 74 (1-2), 99-108 (1999)

11 S. Murad and J. Lin, "Using thin zeolite membranes and external electric fields to separate supercritical aqueous electrolyte solutions," Industrial \& Engineering Chemistry Research 41 (5), 1076-1083 (2002).

12 B. Gong, H. Q. Zeng, J. Zhu et al., "Creating nanocavities of tunable sizes: Hollow helices," Proc. Natl. Acad. Sci. U. S. A. 99 (18), 11583-11588 (2002).

13 C. F. Lopez, S. O. Nielsen, P. B. Moore et al., "Understanding nature's design for a nanosyringe," Proc. Natl. Acad. Sci. U. S. A. 101 (13), 4431-4434 (2004). 
14 O. Beckstein and M. S. P. Sansom, "The influence of geometry, surface character, and flexibility on the permeation of ions and water through biological pores," Physical Biology 1 (1-2), 42-52 (2004).

15 J. L. Barrat and L. Bocquet, "Large slip effect at a nonwetting fluid-solid interface," Phys. Rev. Lett. 82 (23), 4671-4674 (1999).

16 S. Murad and I. K. Puri, "Dynamics of nanoscale jet formation and impingement on flat surfaces," Physics of Fluids 19, 128102 (2007).

17 M. J. Fuerstman, P. Deschatelets, R. Kane, A.Schwartz, , P. J. Kenis, J. M. Deutch, and G. M. Whitesides, Solving mazes using microfluidic networks. Langmuir 19, 4714-4722 (2003).

18 M. P. Allen and D. J. Tildesley, Computer Simulation of Liquids (Clarendon Press, Oxford, 1987).

19 D. J. Evans and S. Murad, "Singularity free algorithm for molecular-dynamics simulation of rigid polyatomics," Molecular Physics 34 (2), 327-331 (1977).

20 I. G. Tironi, R. Sperb, P. E. Smith et al., "A generalized reaction field method for molecular-dynamics simulations," Journal of Chemical Physics 102 (13), 5451-5459 (1995)

21 H. J. C. Berendson, J. Postma, and W. F. van Gunsteren, in Intermolecular Forces, edited by B. Pullman (Reidel, Dordecht, 1981).

22 . Chandrasekhar, S. F. Smith, and W. L. Jorgensen, "SN2 reaction profiles in the gas-phase and aqueous-solution," Journal of the American Chemical Society 106 (10), 3049-3050 (1984).

23 R. O. Watts, "Monte Carlo studies of liquid water," Molecular Physics 28 (4), 1069-1083 (1974).

24 S. Murad and I. K. Puri, "Molecular simulation of thermal transport across hydrophilic interfaces," Chemical Physics Letters 467 (1-3), 110-113 (2008)

25 S. Murad and I. K. Puri, "Thermal transport across nanoscale solid-fluid interfaces," Applied Physics Letters 92, 133105 (2008).

26 U. Rabe, K. Janser, and W. Arnold, "Vibrations of free and surface-coupled atomic force microscope cantilevers: Theory and experiment," Review of Scientific Instruments 67 (9), 3281-3293 (1996). 